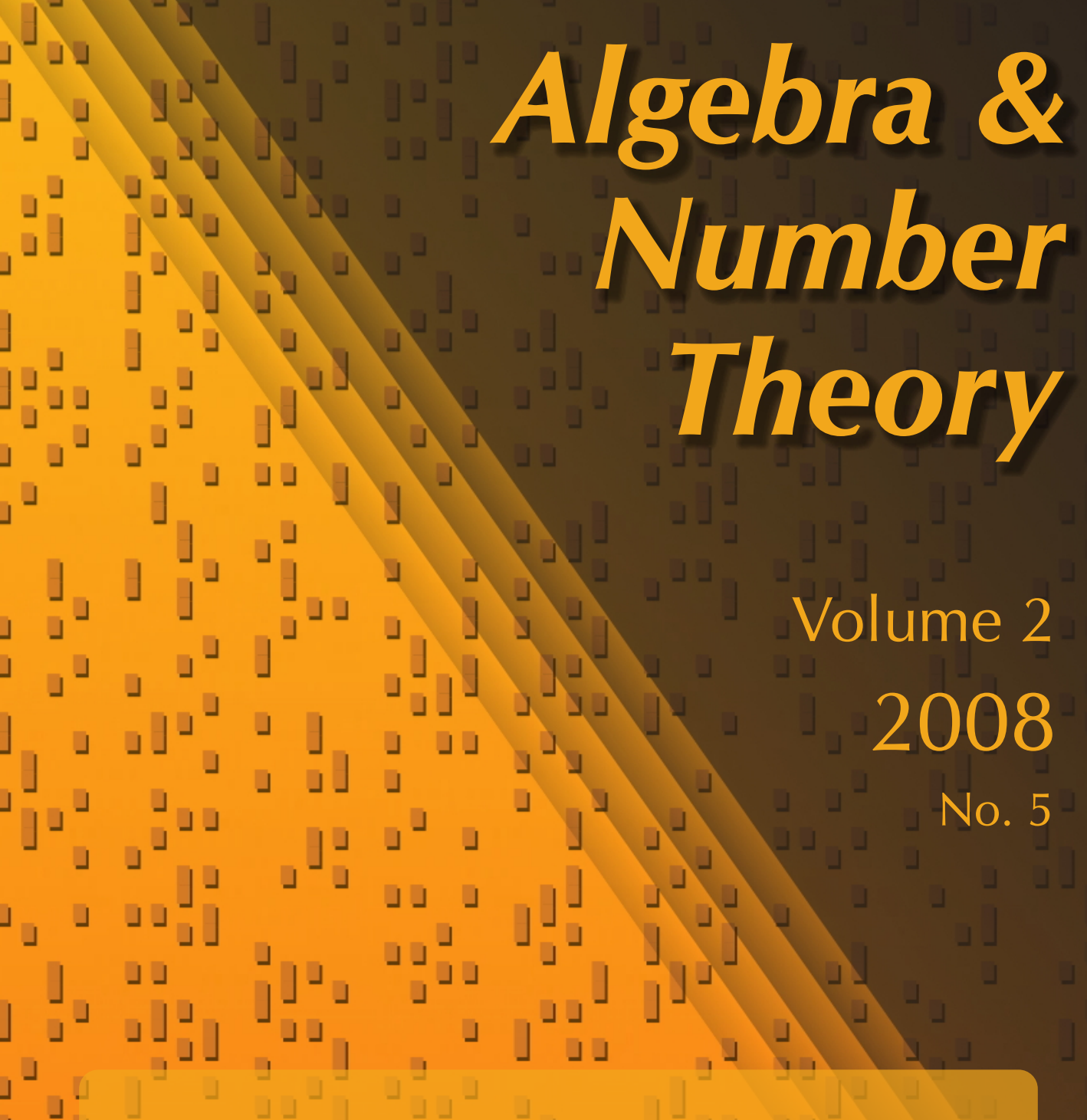

\title{
Constructing simply laced Lie algebras from
}

extremal elements

\lrcorner$\lrcorner$ Jan Draisma and Jos in $t$ panhuis

」」

J $-$

$$
\begin{array}{r}
\text { AVgebra \& } \\
\text { Number } \\
\text { Theory } \\
\text { volume } \\
2008
\end{array}
$$

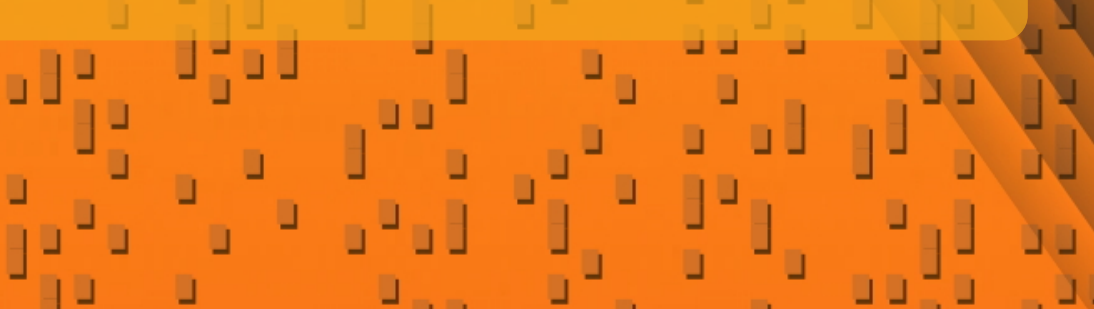

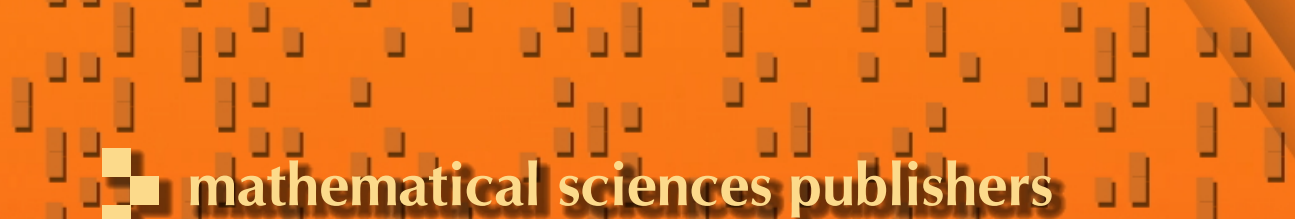

J mathematical sciences publishers 」」 


\title{
Constructing simply laced Lie algebras from extremal elements
}

\author{
Jan Draisma and Jos in 't panhuis
}

\begin{abstract}
For any finite graph $\Gamma$ and any field $K$ of characteristic unequal to 2, we construct an algebraic variety $X$ over $K$ whose $K$-points parametrize $K$-Lie algebras generated by extremal elements, corresponding to the vertices of the graph, with prescribed commutation relations, corresponding to the nonedges. After that, we study the case where $\Gamma$ is a connected, simply laced Dynkin diagram of finite or affine type. We prove that $X$ is then an affine space, and that all points in an open dense subset of $X$ parametrize Lie algebras isomorphic to a single fixed Lie algebra. If $\Gamma$ is of affine type, then this fixed Lie algebra is the split finite-dimensional simple Lie algebra corresponding to the associated finite-type Dynkin diagram. This gives a new construction of these Lie algebras, in which they come together with interesting degenerations, corresponding to points outside the open dense subset. Our results may prove useful for recognizing these Lie algebras.
\end{abstract}

\section{Introduction and main results}

An extremal element of a Lie algebra $\mathscr{L}$ over a field $K$ of characteristic unequal to 2 is an element $x \in \mathscr{L}$ for which $[x,[x, \mathscr{L}]] \subseteq K x$. A sandwich element is an $x \in \mathscr{L}$ satisfying the stronger condition $[x,[x, \mathscr{L}]]=0$. The definition of extremal elements in characteristic 2 is more involved, which is one reason for restricting ourselves to characteristics unequal to 2 here. Extremal elements and sandwich elements play important roles in both classical and modern Lie algebra theory. In complex simple Lie algebras, or their split analogues over other fields, extremal elements are precisely the elements that are long-root vectors relative to some maximal torus. Sandwich elements are used in the classification of simple Lie algebras in small characteristics [Premet and Strade 1997]; they occur in the modular Lie algebras of Cartan type, such as the Witt algebras. Sandwich elements were originally introduced in relation with the restricted Burnside problem [Kostrikin 1981]. An

MSC2000: primary 17B20; secondary 14D20, 17B67, $17 \mathrm{~B} 01$.

Keywords: Lie algebras, extremal elements, generators and relations.

Draisma is supported by DIAMANT, an NWO mathematics cluster. In 't panhuis is supported by NWO PhD grant 10002490. 
important insight for the resolution of this problem is the fact that a Lie algebra generated by finitely many sandwich elements is necessarily finite-dimensional. While this fact was first only proved under extra assumptions, in [Zelmanov and Kostrikin 1990] it is proved in full generality. We will use this result in what follows.

The prominence of extremal elements in the work of Kostrikin and Zel'manov and in modular Lie algebra theory led to the natural problem of describing all Lie algebras generated by a fixed number of extremal elements [Cohen et al. 2001; in 't panhuis et al. 2007; Postma 2007; Roozemond 2005].

Example. Suppose that we want to describe all Lie algebras $\mathscr{L}$ generated by two extremal elements $x$ and $y$. Since $[x,[x, y]]$ is a scalar multiple $a x$ of $x$ and $[y,[x, y]]=-[y,[y, x]]$ is a scalar multiple - by of $y, \mathscr{L}$ is spanned by $x, y,[x, y]$. There may be linear dependencies between these elements, but let us assume that they are linearly independent. Then

$a[y, x]=[y,[x,[x, y]]]=[[y, x],[x, y]]+[x,[y,[x, y]]]=0-b[x, y]=b[y, x]$,

and since we have assumed that $[x, y] \neq 0$, we find that $a=b$. Hence threedimensional Lie algebras with a distinguished pair of extremal generators are parametrized by the single number $a$. Moreover, all algebras with $a \neq 0$ are mutually isomorphic and isomorphic to the split simple Lie algebra of type $A_{1}$, while the algebra with $a=0$ is nilpotent and isomorphic to the three-dimensional Heisenberg algebra. This is a prototypical example of our results. The next smallest case of three generators is treated in [Cohen et al. 2001; Zelmanov and Kostrikin 1990] and also by our results below. There the generic Lie algebra is split of type $A_{2}$ and more interesting degenerations exist.

We now generalize and formalize this example to the case of more generators, where we also allow for the flexibility of prescribing that certain generators commute. Thus let $\Gamma$ be a finite simple graph without loops or multiple edges. Let $\Pi$ be the vertex set of $\Gamma$ and denote the neighbor relation by $\sim$. Fixing a field $K$ of characteristic unequal to 2 , we denote by $\mathscr{F}$ the quotient of the free Lie algebra over $K$ generated by $\Pi$ modulo the relations

$$
[x, y]=0 \quad \text { for all } x, y \in \Pi \text { with } x \ngtr y .
$$

So $\mathscr{F}$ depends both on $\Gamma$ and on $K$, but we will not make this dependence explicit in the notation. We write $\mathscr{F}^{*}$ for the space of all $K$-linear functions $\mathscr{F}_{F} \rightarrow K$. For every $f \in\left(\mathscr{F}^{*}\right)^{\Pi}$, also written $\left(f_{x}\right)_{x \in \Pi}$, we denote by $\mathscr{L}(f)$ the quotient of $\mathscr{F}$ by the ideal $\mathscr{T}(f)$ generated by the (infinitely many) elements

$$
[x,[x, y]]-f_{x}(y) x \quad \text { for } x \in \Pi \text { and } y \in \mathscr{F} .
$$


By construction, $\mathscr{L}(f)$ is a Lie algebra generated by extremal elements, corresponding to the vertices of $\Gamma$, which commute when they are not connected in $\Gamma$. The element $f_{x}$ is a parameter needed to express the extremality of $x \in \Pi$. If $\Gamma$ is not connected, then both $\mathscr{F}$ and $\mathscr{L}(f)$ naturally split into direct sums over all connected components of $\Gamma$, so it is no restriction to assume that $\Gamma$ is connected; we will do so throughout this paper.

In the Lie algebra $\mathscr{L}(0)$, the elements of $\Pi$ map to sandwich elements; hence by [Zelmanov and Kostrikin 1990] this Lie algebra is finite-dimensional. For general $f \in\left(\mathscr{F}^{*}\right)^{\Pi}$ it turns out that $\operatorname{dim} \mathscr{L}(f) \leq \operatorname{dim} \mathscr{L}(0)$; see [Cohen et al. 2001] or the proof of Theorem 1 below. It is therefore natural to focus on the Lie algebras $\mathscr{L}(f)$ of the maximal possible dimension $\operatorname{dim} \mathscr{L}(0)$. This leads us to define the set

$$
X:=\left\{f \in\left(\mathscr{F}^{*}\right)^{\Pi} \mid \operatorname{dim} \mathscr{L}(f)=\operatorname{dim} \mathscr{L}(0)\right\},
$$

the parameter space for all maximal-dimensional Lie algebras of the form $\mathscr{L}(f)$.

Example. In the two-generator case above, $\Gamma$ is the graph with two vertices joined by an edge. The sandwich algebra $\mathscr{L}(0)$ is the three-dimensional Heisenberg algebra, and the condition that $\operatorname{dim} \mathscr{L}(f)=3$ corresponds to our assumption above that $x, y,[x, y]$ be linearly independent. This linear independence forced the parameters $a$ and $b$ to be equal. Here $X$ is the affine line with coordinate $a$. All Lie algebras corresponding to points $a \neq 0$ are mutually isomorphic.

Our first main result is that $X$ carries a natural structure of an affine algebraic variety. To specify this structure we note that $\Phi(0)$ is a homogeneous ideal relative to the natural $\mathbb{N}$-grading that $\mathscr{F}$ inherits from the free Lie algebra generated by $\Pi$.

Theorem 1. The set $X$ is naturally the set of $K$-rational points of an affine variety of finite type defined over $K$. This variety can be described as follows. Fix any finite-dimensional homogeneous subspace $V$ of $\mathscr{F}$ such that $V+\mathscr{T}(0)=\mathscr{F}$. Then the restriction map

$$
X \rightarrow\left(V^{*}\right)^{\Pi}, f \mapsto\left(\left.f_{x}\right|_{V}\right)_{x \in \Pi}
$$

maps $X$ injectively onto the set of $K$-rational points of a closed subvariety of $\left(V^{*}\right)^{\Pi}$. This yields a $K$-variety structure on $X$ which is independent of the choice of $V$.

We prove this theorem in Section 2. In Section 3 we first derive some relations between the sandwich algebra $\mathscr{L}(0)$ and the positive part of the complex KacMoody algebra of type $\Gamma$. Then we determine $\mathscr{L}(0)$ explicitly in the case where $\Gamma$ is a simply laced Dynkin diagram of finite or affine type; by this we mean any of the diagrams in Figure 1 without or with vertex 0, respectively. See Theorems 12 and 13. In Section 4 we study the variety $X$. After some observations for general $\Gamma$, we again specialize to the diagrams of Figure 1 . For these we prove that $X$ is an 


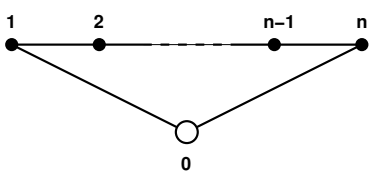

$A_{n}^{(1)}$

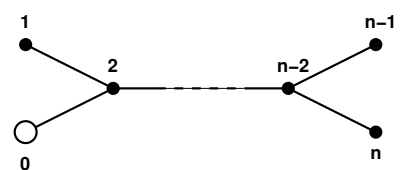

$D_{n}^{(1)}$

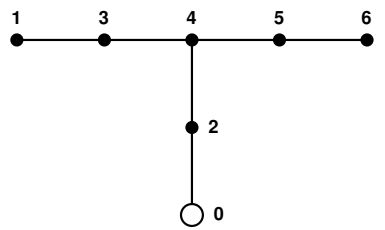

$E_{6}^{(1)}$

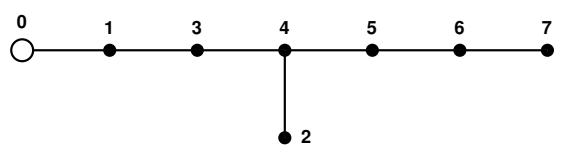

$E_{7}^{(1)}$

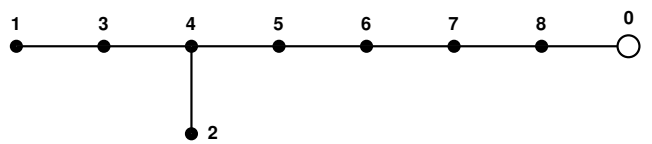

$E_{8}^{(1)}$

Figure 1. The simply laced Dynkin diagrams of affine type. The notation comes from [Kac 1990], and the corresponding finitetype diagrams are obtained by deleting vertex 0 .

affine space, and that for $f$ in an open dense subset of $X$, the Lie algebra $\mathscr{L}(f)$ is isomorphic to a fixed Lie algebra; see Theorems 19 and 22. The latter of these theorems is our second main result, and we paraphrase it here.

Theorem 2. Let $\Gamma$ be any of the simply laced Dynkin diagrams of affine type in Figure 1, let $\Gamma^{0}$ be the finite-type diagram obtained by removing vertex 0 from $\Gamma$, and let $\Sigma$ be the edge set of $\Gamma$. Then $X$ is isomorphic to the affine space of dimension $|\Sigma|+1$ over $K$, and for $f$ in an open dense subset of $X$, the Lie algebra $\mathscr{L}(f)$ is isomorphic to the Chevalley algebra of type $\Gamma^{0}$.

Remark 3. By the Chevalley algebra of type $\Gamma^{0}$ we mean the Lie algebra obtained by tensoring a certain $\mathbb{Z}$-form of the complex simple Lie algebra of type $\Gamma^{0}$ with the field $K$; see Section 3.2 for details. This Lie algebra is often simple, but not always; see [Strade 2004, Chapter 4] and [Seligman 1967].

In Section 5 we will conclude with remarks on applications and related work.

\section{The variety structure of the parameter space}

Recall the notation from Section 1: $\Gamma$ is a connected finite graph without loops or multiple edges, $K$ is a field of characteristic unequal to 2, and $X$ is the set of all $f \in\left(\mathscr{F}^{*}\right)^{\Pi}$ such that $\mathscr{L}(f)$ has the maximal possible dimension, namely that of $\mathscr{L}(0)$. To avoid formulas with many Lie brackets, we write $x_{d} \cdots x_{1}$ for the expression $\left[x_{d},\left[\cdots\left[x_{2}, x_{1}\right] \cdots\right]\right]$. Such an element is called a monomial in the $x_{i}$ of degree $d$. In the proof of Theorem 1 we use the $\mathbb{N}$-grading $\mathscr{F}_{F}=\bigoplus_{d=1}^{\infty} \mathscr{F}_{d}$ of $\mathscr{F}_{F}$, where $\mathscr{F}_{d}$ is the span of all monomials of degree $d$ in the elements of $\Pi$. We also use the following terminology: A subspace $V$ of $\mathscr{F}$ is called homogeneous if it 


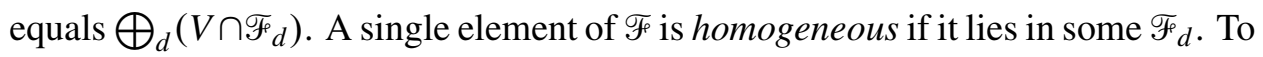
any subspace $V$ of $\mathscr{F}$ we associate the homogeneous subspace gr $V$ of $\mathscr{F}$ spanned by all $v_{d}$ as $v=v_{1}+\cdots+v_{d}$ for $v_{i} \in \mathscr{F}_{i}$ runs through $V$. If $V$ is an ideal, then so is $\operatorname{gr} V$.

Proof of Theorem 1. Let $V$ be a finite-dimensional homogeneous subspace of $\mathscr{F}$ such that $\mathscr{F}=V \oplus \mathscr{I}(0)$; such a subspace exists as $\mathscr{L}(0)$ is finite-dimensional [Cohen et al. 2001; Zelmanov and Kostrikin 1990] and $\mathscr{\Phi}(0)$ is homogeneous. Note that the theorem only requires that $\mathscr{F}=V+\mathscr{(}(0)$; we will argue later why this suffices. Observe that $V$ contains the image of $\Pi$ in $\mathscr{L}$ : the abelian Lie algebra spanned by $\Pi$ is clearly a quotient of $\mathscr{L}(0)$, so the component of $\mathscr{T}(0)$ in degree 1 is trivial. From the shape of the generators (1), it is clear that the homogeneous ideal gr $\mathscr{\Psi}(f)$ associated to $\mathscr{I}(f)$ contains $\mathscr{I}(0)$, so that $\mathscr{F}=V+\mathscr{I}(f)$ for all $f$, and $\mathscr{F}=V \oplus \mathscr{I}(f)$ if and only if $f \in X$. We will argue that the map

$$
\Psi: X \rightarrow\left(V^{*}\right)^{\Pi}, f \mapsto\left(\left.f_{x}\right|_{V}\right)_{x \in \Pi}=:\left.f\right|_{V}
$$

is injective, and that its image is a closed subvariety of $\left(V^{*}\right)^{\Pi}$.

For each $f \in X$, let $\pi_{f}: \mathscr{F}_{P} \rightarrow V$ be the projection onto $V$ along $\Phi(f)$. We prove two slightly technical statements: First, for all $u \in \mathscr{F}$ there exists a polynomial map $P_{u}:\left(V^{*}\right)^{\Pi} \rightarrow V$ such that

$$
P_{u}\left(\left.f\right|_{V}\right)=\pi_{f}(u) \text { for all } f \in X
$$

and second, for $x \in \Pi$ and $u \in \mathscr{F}$ there exists a polynomial $Q_{x, u}:\left(V^{*}\right)^{\Pi} \rightarrow K$ such that

$$
\begin{aligned}
Q_{x, u}\left(\left.f\right|_{V}\right)=f_{x}(u) & \text { for all } f \in X \text { and } \\
Q_{x, u}(h)=h_{x}(u) & \text { if } u \in V \text { and } h \in\left(V^{*}\right)^{\Pi .}
\end{aligned}
$$

We proceed by induction on the degree of $u$ : assume that both statements are true in all degrees less than $d$, and write $u=u_{1}+u_{2}+u_{3}$, where $u_{1}$ has degree less than $d, u_{2} \in V \cap \mathscr{F}_{d}$, and $u_{3} \in \mathscr{I}(0) \cap \mathscr{F}_{d}$. Then $u_{3}$ can be written as a sum of terms of the form $x_{k} \cdots x_{1} x_{1} u^{\prime}$ with $x_{i} \in \Pi$ and $u^{\prime}$ of degree $d-(k+1)<d$. Modulo $\Phi(f)$ for $f \in X$, this term is equal to

$$
f_{x_{1}}\left(u^{\prime}\right) \pi_{f}\left(x_{k} \cdots x_{1}\right)=Q_{x_{1}, u^{\prime}}\left(\left.f\right|_{V}\right) P_{x_{k} \cdots x_{1}}\left(\left.f\right|_{V}\right),
$$

where we used the induction hypothesis for $u^{\prime}$ and $x_{k} \cdots x_{1}$. Hence a $P_{u}$ of the form

$$
P_{u}:=P_{u_{1}}+u_{2}+\text { terms of the form } Q_{x_{1}, u^{\prime}} P_{x_{k} \cdots x_{1}}
$$

has the required property. Similarly, for $x \in \Pi$ and $f \in X$ we have $f_{x}\left(x_{k} \cdots x_{1} x_{1} u^{\prime}\right) x=x x x_{k} \cdots x_{1} x_{1} u^{\prime}=Q_{x_{1}, u^{\prime}}\left(\left.f\right|_{V}\right) Q_{x, x_{k} \cdots x_{1}}\left(\left.f\right|_{V}\right) x \bmod \mathscr{I}(f)$, 
and since $x \notin \mathscr{I}(f)$ we conclude that

$$
f_{x}\left(x_{k} \cdots x_{1} x_{1} u^{\prime}\right)=Q_{x_{1}, u^{\prime}}\left(\left.f\right|_{V}\right) Q_{x, x_{k} \cdots x_{1}}\left(\left.f\right|_{V}\right) .
$$

Hence we may define $Q_{x, u}$ by

$$
Q_{x, u}(h):=Q_{x, u_{1}}(h)+h_{x}\left(u_{2}\right)+\text { terms of the form } Q_{x_{1}, u^{\prime}}(h) Q_{x, x_{k} \cdots x_{1}}(h)
$$

for $h \in\left(V^{*}\right)^{\Pi}$. This shows the existence of $P_{u}$ and $Q_{x, u}$. The injectivity of $\Psi$ is now immediate: any $f \in X$ is determined by its restriction to $V$ by $f_{x}(u)=Q_{x, u}\left(\left.f\right|_{V}\right)$.

We now show that $\operatorname{im}(\Psi)$ is closed. For any tuple $h \in\left(V^{*}\right)^{\Pi}$, one may try to define a Lie algebra structure on $V$ by setting

$$
[u, v]_{h}:=P_{[u, v]}(h) \text { for } u, v \in V .
$$

By construction, if $h=\left.f\right|_{V}$ for some $f \in X$, then this turns $V$ into a Lie algebra isomorphic to $\mathscr{L}(f)$. In this case the Lie bracket has the following two properties:

(i) If $v \in V$ is expressed as a linear combination $\sum_{x_{1}, \ldots, x_{d} \in \Pi} c_{\left(x_{d}, \ldots, x_{1}\right)} x_{d} \cdots x_{1}$ of monomials in the elements of $\Pi$, where the Lie bracket is taken in $\mathscr{F}$, then the expression $\sum_{x_{1}, \ldots, x_{d} \in \Pi} c_{\left(x_{d}, \ldots, x_{1}\right)}\left[x_{d},\left[\cdots\left[x_{2}, x_{1}\right]_{h} \cdots\right]_{h}\right]_{h}$ also equals $v$; and

(ii) $\left[x,[x, u]_{h}\right]_{h}=Q_{x, u}(h) x$ for all $x \in \Pi$ and $u \in V$.

Conversely, suppose that $[\cdot, \cdot]_{h}$ indeed defines a Lie algebra on $V$ satisfying (i) and (ii). Then $\left(V,[\cdot, \cdot]_{h}\right)$ is a Lie algebra of dimension $\operatorname{dim} \mathscr{L}(0)$ that by (i) is generated by the image of $\Pi$, and by (ii) this image consists of extremal elements. Hence there exists an $f \in X$ corresponding to this Lie algebra, and its restriction to $V$ is $h$-indeed, $f_{x}(u)$ is the coefficient of $x$ in $\left[x[x, u]_{h}\right]_{h}$, which is $Q_{x, u}(h)=$ $h_{x}(u)$ for $u \in V$. Finally, all stated conditions on $h$ - the fact that $[\cdot, \cdot]_{h}$ satisfies the Jacobi identity and anticommutativity, together with (i) and (ii) — are closed; here we use the polynomiality of $P_{u}$ and $Q_{x, u}$. This proves that im( $(\Psi)$ is closed.

Now if $U$ is any homogeneous subspace containing $V$, then the restriction map $\Psi^{\prime}: X \rightarrow\left(U^{*}\right)^{\Pi}$ is clearly also injective. Moreover, an $h^{\prime} \in\left(U^{*}\right)^{\Pi}$ lies in the image of this map if and only if $\left.h^{\prime}\right|_{V}$ lies in im $\Psi$ and $h_{x}^{\prime}(u)=Q_{x, u}\left(\left.h^{\prime}\right|_{V}\right)$ for all $u \in U$. Thus $\operatorname{im} \Psi^{\prime}$ is closed, and the maps $\operatorname{im} \Psi^{\prime} \rightarrow \operatorname{im} \Psi,\left.h^{\prime} \mapsto h^{\prime}\right|_{V}$ and $\operatorname{im} \Psi \rightarrow \operatorname{im} \Psi^{\prime}, h \mapsto h^{\prime}$ with $h_{x}^{\prime}(u)=Q_{x, u}(h)$ for $u \in U$ are inverse morphisms between im $\Psi$ and im $\Psi^{\prime}$. Similarly, if $V^{\prime}$ is any other homogeneous vector space complement of $\mathscr{}(0)$ contained in $U$, then the restriction map $\left(U^{*}\right)^{\Pi} \rightarrow\left(\left(V^{\prime}\right)^{*}\right)^{\Pi}$ induces an isomorphism between the images of $X$ in these spaces. This shows that the variety structure of $X$ does not depend on the choice of $V$. Finally, all morphisms indicated here are defined over $K$. We conclude that we have a $K$ variety structure on $X$ that is independent of the choice of $V$.

The type of reasoning in this proof will return in Section 4: in the case where $\Gamma$ is a Dynkin diagram, we will show that for $f \in X$ the restriction $\left.f\right|_{V}$ actually depends 
polynomially on even fewer values of the $f_{x}$, thus embedding $X$ into smaller affine spaces. That these embeddings are closed can be proved exactly as we did above.

\section{The sandwich algebra}

For now, $\Gamma$ is an arbitrary finite graph (not necessarily a Dynkin diagram). The Lie algebra $\mathscr{L}(0)$ is the so-called sandwich algebra corresponding to $\Gamma$. It is a finite-dimensional nilpotent Lie algebra, and carries an $\mathbb{N}^{\Pi}$-grading defined as follows. The weight of a word $\left(x_{d}, \ldots, x_{1}\right)$ over $\Pi$ is the element $\mu \in \mathbb{N}^{\Pi}$ whose $x$-coordinate equals

$$
\left|\left\{i \in\{1, \ldots, d\} \mid x_{i}=x\right\}\right|
$$

for all $x \in \Pi$. For such a word the corresponding monomial $x_{d} \cdots x_{1}$ lives in the free Lie algebra on $\Pi$, but we use the same notation for its images in $\mathscr{F}$ and $\mathscr{L}(f)$ when this does not lead to any confusion. We will sometimes say that a monomial $x_{d} \cdots x_{1} \in \mathscr{L}(0)$ has weight $\mu$, by which we mean that the word $\left(x_{d}, \ldots, x_{1}\right)$ has weight $\mu$ - the monomial $x_{d} \cdots x_{1}$ itself might be 0 . Now the free Lie algebra is graded by weight, and this grading refines the grading by degree. Like the grading by degree, the grading by weight is inherited by $\mathscr{L}(0)$ as all relations defining $\mathscr{L}(0)$ are monomials. We write $\mathscr{L}(0)_{\mu}$ for the space of weight $\mu \in \mathbb{N}^{\Pi}$ and call $\operatorname{dim} \mathscr{L}(0)_{\mu}$ the multiplicity of $\mu$.

For $x \in \Pi$, let $\alpha_{x}$ be the element with a 1 on position $x$ and zeros elsewhere; that is, $\alpha_{x}$ is the weight of the word $(x)$. We define a symmetric $\mathbb{Z}$-bilinear form $\langle\cdot, \cdot\rangle$ on $\mathbb{Z}^{\Pi}$ by its values on the standard basis: for $x, y \in \Pi$ we set

$$
\left\langle\alpha_{x}, \alpha_{y}\right\rangle:=\left\{\begin{aligned}
2 & \text { if } x=y, \\
-1 & \text { if } x \sim y, \text { and } \\
0 & \text { otherwise. }
\end{aligned}\right.
$$

The matrix $A:=\left(\left\langle\alpha_{x}, \alpha_{y}\right\rangle\right)_{x, y \in \Pi}$ is called the Cartan matrix of $\Gamma$. The height of an element of $\mathbb{Z}^{\Pi}$ is by definition the sum of the coefficients of the $\alpha_{x}$ in it for $x \in \Pi$.

In what follows we often need to show that certain monomials $x_{d} \cdots x_{1}$ are zero in $\mathscr{L}(0)$. Lemmas 6-10 show how the bilinear form comes into play. But first we recall an elementary property of sandwich elements, to which they owe their name.

Lemma 4. Let $x$ be a sandwich element in a Lie algebra $\mathscr{L}$, and let $y, z \in \mathscr{L}$ be arbitrary. Then $x y x z=0$.

Proof. We have

$$
\begin{aligned}
x y x z & =[x, y] x z+y x x z=[x, y] x z=-[x, x y] z+x[x, y] z \\
& =z x x y+x[x, y] z=x[x, y] z=x x y z-x y x z=-x y x z .
\end{aligned}
$$


Remark 5. Note that we have used here that the characteristic is not 2 . In the case of characteristic 2, this lemma should be taken as part of the definition of a sandwich element [Cohen and Ivanyos 2006].

Lemma 6. Let $w=\left(x_{d}, x_{d-1}, \ldots, x_{1}\right)$ be a word over $\Pi$, and let $x \in \Pi$. Let $x_{i}$ and $x_{j}$ be consecutive occurrences of $x$ in $w$ (that is, $i>j, x_{i}=x_{j}=x$, and $x_{k} \neq x$ for all $k$ strictly between $i$ and $j$ ). Suppose that the letters in $w$ strictly between $x_{i}$ and $x_{j}$ contain at most 1 occurrence of a $\Gamma$-neighbor of $x$, that is, the set $\left\{k \in\{j+1, \ldots, i-1\} \mid x_{k} \sim x\right\}$ has cardinality at most 1 . Then $x_{d} x_{d-1} \cdots x_{1}$ is 0 in $\mathscr{L}(0)$.

Proof. Set $z:=x_{d} x_{d-1} \cdots x_{1}$. First, using the fact that on $\mathscr{F}$ the linear map $\operatorname{ad}(x)$ commutes with $\operatorname{ad}(y)$ for any $y \in \Pi$ with $x \neq y$, we can move $x_{i}$ in $z$ to the right until it is directly to the left of either $x_{j}$ or the unique $x_{k} \sim x$ between $x_{i}$ and $x_{j}$, so we may assume that this was already the case to begin with.

If $i=j+1$ then either $j=1$ and $z$ is zero by anticommutativity, or $j>1$ and the monomial $x_{i} x_{j} x_{j-1} \cdots x_{1}=x x x_{j-1} \cdots x_{1}$ is zero by the sandwich property of $x$.

Suppose, on the other hand, that $x_{i} \sim x_{i-1}$. Then if $j=1$ and $i-1>2$, the monomial $z$ is zero since $x_{2} x_{1}$ is - indeed, $x_{2} \not x_{1}$. On the other hand, if $i-1=2$ or $j>1$, then we can move $x_{j}$ in $z$ to the left until it is directly to the right of $x_{i-1}$. So again, we may assume that it was there from the beginning. But now

$$
x_{i} x_{i-1} x_{j} x_{j-1} \cdots x_{1}=x x_{i-1} x x_{j-1} \cdots x_{1} \text {. }
$$

If $j>1$, then this monomial equals zero by Lemma 4 ; and if $j=1$, then it is zero by the sandwich property of $x$.

Lemma 7. Let $\left(x_{d}, x_{d-1}, \ldots, x_{1}\right)$ be a word with $d \geq 2$ over $\Pi$, and suppose that the weight $\mu$ of $\left(x_{d-1}, x_{d-2}, \ldots, x_{1}\right)$ satisfies $\left\langle\alpha_{x_{d}}, \mu\right\rangle \geq 0$. Then $x_{d} x_{d-1} \cdots x_{1}=0$ in $\mathscr{L}(0)$.

Proof. Set $w:=\left(x_{d}, x_{d-1}, \ldots, x_{1}\right)$ and $z:=x_{d} x_{d-1} \cdots x_{1}$. The condition on the bilinear form can be written as

$$
2\left|\left\{j \in\{1, \ldots, d-1\} \mid x_{j}=x_{d}\right\}\right| \geq\left|\left\{j \in\{1, \ldots, d-1\} \mid x_{j} \sim x_{d}\right\}\right| .
$$

First we note that if the right side is 0 , then $z$ is trivially zero: then all $x_{i}$ with $i<d$ commute with $x_{d}$, and there at least $d-1 \geq 1$ such factors. So we may assume that the right side is positive, and hence so is the left side.

Let the set in the left side of this inequality consist of the indices $i_{m}>i_{m-1}>$ $\cdots>i_{1}$. By the above, $m$ is positive. In the word $w$ there are $m$ pairs $(i, j)$ satisfying the conditions of Lemma 6 with $x=x_{d}$, namely, $\left(d, i_{m}\right),\left(i_{m}, i_{m-1}\right), \ldots$, $\left(i_{2}, i_{1}\right)$. Now if for some such $(i, j)$ there are less than two $\Gamma$-neighbors of $x_{d}$ in the interval between $x_{i}$ and $x_{j}$, then $z=0$ by Lemma 6 . So we may assume that each of these $m$ intervals contains at least two $\Gamma$-neighbors of $x_{d}$. But then, by the 
inequality above, these exhaust all $\Gamma$-neighbors of $x_{d}$ in $w$, so in particular there are exactly $2 \Gamma$-neighbors of $x_{d}$ between $x_{i_{2}}$ and $x_{i_{1}}$, and none to the right of $x_{i_{1}}$. Now if $i_{1}>1$, then $z$ is zero because $x_{i_{1}}$ commutes with everything to the right of it. Hence assume that $i_{1}=1$, and note that $i_{2} \geq 4$. If $x_{2} \nsucc x_{1}=x_{i_{1}}$, then again $z$ is trivially 0 , so assume that $x_{2}$ is a $\Gamma$-neighbor of $x_{d}=x_{1}$. Then we have

$$
x_{i_{2}} \cdots x_{3} x_{2} x_{1}=-x_{i_{2}} \cdots x_{3} x_{1} x_{2}
$$

but in the monomial on the right there is only one $\Gamma$-neighbor of $x_{d}$ between $x_{i_{2}}$ and $x_{1}$ - hence it is zero by Lemma 6 .

Lemma 8. Let $x \in \Pi$ and $\lambda \in \mathbb{N}^{\Pi}$ satisfy $\left\langle\alpha_{x}, \lambda\right\rangle=-1$. Then $\mathscr{L}(0)_{\alpha_{x}+\lambda}=$ $\left[x, \mathscr{L}(0)_{\lambda}\right]$.

Proof. Let $w=\left(x_{d}, \ldots, x_{1}\right)$ be a word over $\Pi$ of weight $\alpha_{x}+\lambda$. We show that in $\mathscr{L}(0)$ the monomial $z:=x_{d} \cdots x_{1}$ is a scalar multiple of some monomial of the form $x z^{\prime}$, where $z^{\prime}$ is a monomial of weight $\lambda$. Obviously, $x$ occurs in $w$; let $k$ be maximal with $x_{k}=x$. If $k=1$, then we may interchange $x_{k}=x_{1}$ and $x_{k+1}=x_{2}$ in $z$ at the cost of a minus sign (note that $d \geq 2$ ), so we may assume that $k \geq 2$.

Suppose first that there occur $\Gamma$-neighbors of $x=x_{k}$ to the left of $x_{k}$ in $w$. We claim that then $z=0$. Indeed, let $\mu$ and $v$ be the weights of $\left(x_{k-1}, \ldots, x_{1}\right)$ and $\left(x_{d}, \ldots, x_{k+1}\right)$, respectively. Then we have

$$
\left\langle\alpha_{x}, \mu\right\rangle=\left\langle\alpha_{x}, \lambda\right\rangle-\left\langle\alpha_{x}, \nu\right\rangle=-1-\left\langle\alpha_{x}, v\right\rangle \geq 0,
$$

where in the last inequality we use that there are occurrences of neighbors of $x_{k}$, but none of $x_{k}$ itself, in the word $\left(x_{d}, \ldots, x_{k+1}\right)$. Now we find $x_{k} \cdots x_{1}=0$ by Lemma 7 (note that $k \geq 2$ ); hence $z=0$ as claimed.

So we can assume that there are no $\Gamma$-neighbors of $x_{k}$ to the left of $x_{k}$ in $w$. Then we may move $x_{k}$ in $z$ all the way to the left; hence $z$ is indeed equal to $x z^{\prime}$ for some monomial $z^{\prime}$ of weight $\lambda$.

3.1. Relation with the root system of the Kac-Moody algebra. Recall the definition of the Kac-Moody algebra $\mathfrak{g}_{\mathrm{KM}}$ over $\mathbb{C}$ corresponding to $\Gamma$ : it is the Lie algebra generated by $3 \cdot|\Pi|$ generators, denoted $E_{x}, H_{x}, F_{x}$ for $x \in \Pi$, modulo the relations

$$
\begin{aligned}
& H_{x} H_{y}=0, \quad E_{x} F_{x}=H_{x}, \\
& H_{x} E_{y}=\left\langle\alpha_{x}, \alpha_{y}\right\rangle E_{y}, \quad H_{x} F_{y}=-\left\langle\alpha_{x}, \alpha_{y}\right\rangle F_{y} \text {; } \\
& \text { and, for } x \neq y, E_{x} F_{y}=0 \text {, } \\
& \operatorname{ad}\left(E_{x}\right)^{1-\left\langle\alpha_{x}, \alpha_{y}\right\rangle} E_{y}=0 \text {, } \\
& \operatorname{ad}\left(F_{x}\right)^{1-\left\langle\alpha_{x}, \alpha_{y}\right\rangle} F_{y}=0 .
\end{aligned}
$$

Endow $\mathfrak{g}_{\mathrm{KM}}$ with the $\mathbb{Z}^{\Pi}$-grading in which $E_{x}, H_{x}, F_{x}$ have weights $\alpha_{x}, 0,-\alpha_{x}$, respectively. Let $\Phi:=\left\{\beta \in \mathbb{Z}^{\Pi} \backslash\{0\} \mid\left(\mathfrak{g}_{\mathrm{KM}}\right)_{\beta} \neq 0\right\}$ be the root system of $\mathfrak{g}_{\mathrm{KM}}$; it is 
equal to the disjoint union of its subsets $\Phi_{ \pm}:=\Phi \bigcap( \pm \mathbb{N})^{\Pi}$ and contains the simple roots $\alpha_{x}$ for $x \in \Pi$; we refer to [Kac 1990] for the theory of Kac-Moody algebras. In what follows we will compare the multiplicities of weights in the $K$-algebra $\mathscr{L}(0)$ and the $\mathbb{C}$-algebra $\mathfrak{g}_{\mathrm{KM}}$.

Lemma 9. For $\lambda \in \mathbb{N}^{\Pi} \backslash \Phi_{+}$, we have $\mathscr{L}(0)_{\lambda}=0$.

Proof. We proceed by induction on the height of $\lambda$. The proposition is trivially true for $\lambda$ of height 1 . Suppose now that it is true for height $d-1 \geq 1$, and consider a word $w=\left(x_{d}, x_{d-1}, \ldots, x_{1}\right)$ of weight $\lambda \notin \Phi_{+}$.

Set $\mu:=\lambda-\alpha_{x_{d}}$. If $\mu \notin \Phi_{+}$, then $x_{d-1} \cdots x_{1}=0$ by the induction hypothesis, so we may assume that $\mu \in \Phi_{+}$. This together with $\mu+\alpha_{x_{d}} \notin \Phi_{+}$implies (by elementary $\mathfrak{s l}_{2}$-theory in $\mathfrak{g}_{\mathrm{KM}}$ ) that $\left\langle\alpha_{x_{d}}, \mu\right\rangle \geq 0$. Now Lemma 7 shows that $x_{d} \cdots x_{1}=0$.

For another relation between weight multiplicities in $\mathscr{L}(0)$ and $\mathfrak{g}_{\mathrm{KM}}$, recall that a root in $\Phi$ is called real if it is in the orbit of some simple root under the Weyl group $W$ of $\mathfrak{g}_{\mathrm{KM}}$. In that case it has multiplicity 1 in $\mathfrak{g}_{\mathrm{KM}}$. We now call a root $\beta \in \Phi_{+}$very real - this is nonstandard terminology - if it can be written as $\beta=$ $\alpha_{x_{d}}+\cdots+\alpha_{x_{1}}$, for some $x_{1}, \ldots, x_{d} \in \Pi$, such that for all $i=2, \ldots, d$ we have

$$
\left\langle\alpha_{x_{i}}, \alpha_{x_{i-1}}+\cdots+\alpha_{x_{1}}\right\rangle=-1 .
$$

(This implies that $\beta=s_{x_{d}} \cdots s_{x_{2}} \alpha_{x_{1}}$, where the $s_{x}$ are the fundamental reflections corresponding to the $x \in \Pi$.)

Lemma 10. Any very real $\beta \in \Phi_{+}$has multiplicity at most 1 in $\mathscr{L}(0)$.

Proof. This follows by induction on the height of $\beta$, using Lemma 8 for the induction step.

3.2. Simply laced Dynkin diagram of finite type. In this section we assume that $\Gamma$ is a Dynkin diagram of finite type, that is, one of the diagrams in Figure 1 with vertex 0 removed. Then $\mathfrak{g}_{\mathrm{KM}}$ is a finite-dimensional simple Lie algebra over $\mathbb{C}$. Now $\mathfrak{g}_{\mathrm{KM}}$ has a Chevalley basis [Carter 1972, Section 4.2]. This basis consists of the images of the $H_{x}$ and one vector $E_{\alpha} \in\left(\mathfrak{g}_{\mathrm{KM}}\right)_{\alpha}$ for every root $\alpha \in \Phi$, where $E_{\alpha_{x}}$ and $E_{-\alpha_{x}}$ may be taken as $E_{x}$ and $F_{x}$, respectively. An important property that we will need is that $\left[E_{\alpha}, E_{\beta}\right]= \pm E_{\alpha+\beta}$ for all roots $\alpha$ and $\beta$ such that $\alpha+\beta$ is a root; here we use that the $p$ in [Carter 1972, Theorem 4.2.1] is 0 in the simply laced case. The Chevalley basis spans a $\mathbb{Z}$-subalgebra of $\mathfrak{g}_{\mathrm{KM}}$. Let $\mathfrak{g}$ be the $K$-algebra obtained by tensoring this $\mathbb{Z}$-form with $K$, and let $E_{x}^{0}, H_{x}^{0}, F_{x}^{0}$ be the images in $\mathfrak{g}$ of $E_{x}, H_{x}, F_{x}$. The Lie algebra $\mathfrak{g}$ has a triangular decomposition

$$
\mathfrak{g}=\mathfrak{n}_{-} \oplus \mathfrak{h} \oplus \mathfrak{n}_{+},
$$

where $\mathfrak{n}_{ \pm}:=\bigoplus_{\beta \in \Phi_{ \pm}} \mathfrak{g}_{\beta}$. We will refer to $\mathfrak{g}$ as the Chevalley algebra of type $\Gamma$. 
Remark 11. One can also define $\mathfrak{g}$ as the Lie algebra of the split simply connected algebraic group over $K$ of type $\Gamma$.

Theorem 12. Let $\Gamma$ be a simply laced Dynkin diagram of finite type, obtained from a diagram in Figure 1 by removing vertex 0 . Let $\mathfrak{g}$ be the corresponding Chevalley algebra over the field $K$ of characteristic unequal to 2 , and let $\mathfrak{n}_{+}$be the subalgebra generated by the $E_{x}^{0}$. Then the map sending $x \in \Pi$ to $E_{x}^{0}$ induces a (necessarily unique) isomorphism $\mathscr{L}(0) \rightarrow \mathfrak{n}_{+}$.

In the proof of this theorem we use the following well-known facts about simply laced Kac-Moody algebras of finite type: first, $\langle\cdot, \cdot\rangle$ only takes the values $-1,0,1,2$ on $\Phi_{+} \times \Phi_{+}$, and second, all roots in $\Phi_{+}$are very real.

Proof of Theorem 12. To prove the existence of a homomorphism $\pi$ sending $x$ to $E_{x}^{0}$, we verify that the relations defining $\mathscr{L}(0)$ hold in $\mathfrak{n}_{+}$. That is, we have to prove that

$$
\begin{aligned}
{\left[E_{x}^{0}, E_{y}^{0}\right]=0 } & \text { for all } x, y \in \Pi \text { with } x \ngtr y, \\
\operatorname{ad}\left(E_{x}^{0}\right)^{2} z=0 & \text { for all } x \in \Pi \text { and all } z \in \mathfrak{n}_{+} .
\end{aligned}
$$

The first statement is immediate from the relations defining $\mathfrak{g}_{\mathrm{KM}}$. For the second relation, if $z \in \mathfrak{n}_{+}$is a root vector with $\operatorname{root} \beta \in \Phi_{+}$, then $\left\langle\beta, \alpha_{x}\right\rangle \geq-1$ by the above, so that $\left\langle\beta+2 \alpha_{x}, \alpha_{x}\right\rangle \geq 3$, and therefore $\beta+2 \alpha_{x} \notin \Phi_{+}$, so that $\operatorname{ad}\left(E_{x}\right)^{2} z=0$. As root vectors span $\mathfrak{n}_{+}$, we have proved the existence of $\pi$; uniqueness is obvious.

Now we have to show that $\pi$ is an isomorphism. It is surjective since $\mathfrak{n}_{+}$is generated by the $E_{x}^{0}$; this follows from the properties of the Chevalley basis in Section 3.2. Hence it suffices to prove that $\operatorname{dim} \mathscr{L}(0) \leq \operatorname{dim} \mathfrak{n}_{+}$. But by Lemmas 9 and 10 and the fact that all roots are very real, we have $\mathscr{L}(0)_{\mu}=0$ for all $\mu \notin \Phi_{+}$ and $\operatorname{dim} \mathscr{L}(0)_{\beta} \leq \operatorname{dim} \mathfrak{g}_{\beta}$ for all $\beta \in \Phi_{+}$. This concludes the proof.

3.3. Simply laced Dynkin diagrams of affine type. Suppose now that $\Gamma$ is a simply laced Dynkin diagram of affine type from Figure 1. Recall that the Cartan matrix $A$ has a one-dimensional kernel spanned by a unique primitive vector $\delta \in \mathbb{N} \Pi$. Here primitive means that the greatest common divisor of the coefficients of $\delta$ on the standard basis is 1 ; indeed, there always exists a vertex $x_{0} \in \Pi$ (labeled 0 in Figure 1) with coefficient 1 in $\delta$, and all such vertices form an $\operatorname{Aut}(\Gamma)$-orbit. For later use, we let $h$ be the Coxeter number, which is the height of $\delta$.

Let $\Pi^{0}:=\Pi \backslash\left\{x_{0}\right\}$. Write $\Gamma^{0}$ for the induced subgraph on $\Pi^{0}$ (which is a Dynkin diagram of finite type) and $\Phi^{0}$ for the root system of the Chevalley algebra $\mathfrak{g}$ of type $\Gamma^{0}$ defined in Section 3.2. This root system lives in the space $\mathbb{Z}^{\Pi^{0}}$, which we identify with the elements of $\mathbb{Z}^{\Pi}$ that are zero on $x_{0}$. Retain the notation $\mathfrak{n}_{ \pm} \subseteq \mathfrak{g}$ from Section 3.2. Consider the semidirect product $\mathfrak{u}:=\mathfrak{n}_{+} \ltimes \mathfrak{g} / \mathfrak{n}_{+}$, where the second summand is endowed with the trivial Lie bracket and the natural $\mathfrak{n}_{+^{-}}$ module structure. This $\mathfrak{u}$ is clearly a nilpotent Lie algebra; we will prove that it 
is isomorphic to $\mathscr{L}(0)$. In our proof we use the following $\mathbb{Z}^{\Pi}$-grading of $\mathfrak{u}$ : the root spaces in $\mathfrak{n}_{+}$have their usual weight in $\Phi_{+}^{0} \subseteq \mathbb{Z}^{\Pi^{0}}$, while the image of $\mathfrak{g}_{\beta}$ in $\mathfrak{g} / \mathfrak{n}_{+} \subseteq \mathfrak{u}$ for $\beta \in\{0\} \cup \Phi_{-}^{0}$ has weight $\delta+\beta$. Thus the set of all weights occurring in $\mathfrak{u}$ is

$$
\Theta:=\Phi_{+}^{0} \bigcup\left\{\delta+\beta \mid \beta \in \Phi_{-}^{0}\right\} \bigcup\{\delta\} .
$$

Theorem 13. Let $\Gamma$ be a simply laced Dynkin diagram of affine type from Figure 1, let $\Gamma^{0}$ be the subdiagram of finite type obtained by removing vertex 0 , and let $\mathfrak{g}$ be the Chevalley algebra of type $\Gamma^{0}$ over a field of characteristic unequal to 2 . For $x \in \Pi^{0}$, let $E_{x}^{0} \in \mathfrak{n}_{+}$be the element of the Chevalley basis of $\mathfrak{g}$ with simple root $\alpha_{x}$, and for the lowest root $\theta \in \Phi_{-}^{0}$, let $E_{\theta}^{0} \in \mathfrak{g} / \mathfrak{n}_{+}$be the image of the element in the Chevalley basis of weight $\theta$. Then the map sending $x \in \Pi^{0}$ to $E_{x}^{0}$ and $x_{0}$ to $E_{\theta}^{0}$ induces a $\mathbb{Z}^{\Pi}$-graded isomorphism $\mathscr{L}(0) \rightarrow \mathfrak{n}_{+} \ltimes \mathfrak{g} / \mathfrak{n}_{+}$of Lie algebras.

Remark 14. Over $\mathbb{C}$ one can argue directly in the Kac-Moody algebra $\mathfrak{g}_{\mathrm{KM}}$. Then $\mathscr{L}(0)$ is also the quotient of the positive nilpotent subalgebra of $\mathfrak{g}_{\mathrm{KM}}$ by the root spaces with roots of height larger than the Coxeter number $h$. In the proof one uses the root multiplicities of [Kac 1990, Proposition 6.3]. One might also pursue this approach in positive characteristic using the results of [Billig 1990], but we have chosen to avoid defining the Kac-Moody algebra in arbitrary characteristic and use the Chevalley basis instead.

Proof of Theorem 13. The proof is close to that of Theorem 12. We start by verifying that the relations defining $\mathscr{L}(0)$ hold in $\mathfrak{u}=\mathfrak{n}_{+} \ltimes \mathfrak{g} / \mathfrak{n}_{+}$. First, $E_{x}^{0}$ and $E_{y}^{0}$ with $x, y \in \Pi^{0}$ commute when they are not connected in $\Gamma$; this follows from the defining equations of $\mathfrak{g}_{\mathrm{KM}}$. Second, $E_{x}^{0}$ and $E_{\theta}^{0}$ commute if $x \in \Pi^{0}$ is not connected to $x_{0}$, as $\theta+\alpha_{x}$ is then not in $\Phi^{0}$. Third, each $E_{x}^{0}$ is a sandwich element in $\mathfrak{u}$ : for its action on $\mathfrak{n}_{+}$, this follows as in the proof of Theorem 12, and for its action on $\mathfrak{g} / \mathfrak{n}_{+}$, it follows from the fact that $\operatorname{ad}\left(E_{x}^{0}\right)^{2} \mathfrak{g} \subseteq K E_{x}^{0} \subseteq \mathfrak{n}_{+}$. Fourth, $E_{\theta}^{0}$ is a sandwich element as $\operatorname{ad}\left(E_{\theta}^{0}\right)$ maps $\mathfrak{u}$ into $\mathfrak{g} / \mathfrak{n}_{+}$, which has trivial multiplication. This shows the existence of a homomorphism $\pi: \mathscr{L}(0) \rightarrow \mathfrak{u}$. Moreover $\pi$ is graded; in particular, the weight of $E_{\theta}^{0}$ is $\delta+\theta=\alpha_{x_{0}}$.

The $E_{x}^{0}$ generate $\mathfrak{n}_{+}$, and $E_{\theta}^{0}$ generates the $\mathfrak{n}_{+}$-module $\mathfrak{g} / \mathfrak{n}_{+}$. These statements follow from properties of the Chevalley basis in Section 3.2, and imply that $\pi$ is surjective. So we need only show that $\operatorname{dim} \mathscr{L}(0) \leq \operatorname{dim} \mathfrak{u}$; we prove this for each weight in $\Theta$.

First, the roots in $\Phi^{0}$ are very real, so their multiplicities in $\mathscr{L}(0)$ are at most 1 by Lemma 10. Second, we claim that all roots of the form $\delta+\beta$ with $\beta \in \Phi_{-}^{0}$ are also very real. This follows by induction on the height of $\beta$ : For $\beta=\theta$ it is clear since $\delta+\theta=\alpha_{x_{0}}$. For $\beta \neq \theta$ it is well known that there exists an $x \in \Pi^{0}$ such that $\left\langle\alpha_{x}, \beta\right\rangle=1$. Then we have $\delta+\beta=\left(\delta+\beta-\alpha_{x}\right)+\alpha_{x}$, where $\delta+\beta-\alpha_{x} \in \Theta$ and $\left\langle\alpha, \delta+\beta-\alpha_{x}\right\rangle=0+1-2=-1$; here we use that $\delta$ is in the radical of the 
form $\langle\cdot, \cdot\rangle$. By induction, $\delta+\beta-\alpha_{x}$ is very real; hence so is $\delta+\beta$. This shows that also the roots of the form $\delta+\beta$ with $\beta \in \Phi_{-}^{0}$ have multiplicity at most 1 in $\mathscr{L}(0)$, again by Lemma 10 .

Next we show that $\delta$ has multiplicity at most $\left|\Pi^{0}\right|=\operatorname{dim} \mathfrak{h}$ in $\mathscr{L}(0)$. Indeed, we claim that $\mathscr{L}(0)_{\delta}$ is contained in $\sum_{x \in \Pi^{0}}\left[x, \mathscr{L}(0)_{\delta-\alpha_{x}}\right]$. Then, by the above, each of the summands has dimension at most 1 , and we are done. The claim is true almost by definition: any monomial of weight $\delta$ must start with some $x \in \Pi$, so we need only show that monomials starting with $x_{0}$ are already contained in the sum above. Consider any monomial $z:=x_{d} \cdots x_{1}$ of weight $\delta$, where $x_{d}=x_{0}$. As the coefficient of $x_{0}$ in $\delta$ is 1 , none of the $x_{i}$ with $i<d$ is equal to $x_{0}$. But then an elementary application of the Jacobi identity and induction shows that $z$ is a linear combination of monomials that do not start with $x_{0}$.

Finally, we have to show that $\mathscr{L}(0)_{\mu}$ is 0 for $\mu \in \Phi_{+} \backslash \Theta$ (we already have $\mathscr{L}(0)_{\mu}=0$ for $\mu \notin \Phi_{+}$by Lemma 9). But Lemma 7 and the fact that $\left\langle\alpha_{x}, \delta\right\rangle=0$ for all $x \in \Pi$ together imply that $\left[x, \mathscr{L}(0)_{\delta}\right]=0$ for all $x \in \Pi$. So it suffices to show that if $\mu \in \Phi_{+}$is not in $\Theta$, then " $\mu$ can only be reached through $\delta$." More precisely, if $\left(x_{d}, \ldots, x_{1}\right)$ is any word over $\Pi$ such that $\sum_{j=1}^{d} \alpha_{x_{j}}=\mu$ and $\mu_{i}:=\sum_{j=1}^{i} \alpha_{x_{j}} \in \Phi_{+}$for all $i=1, \ldots, d$, then there exists an $i$ such that $\mu_{i}=\delta$. But this follows immediately from the fact that $\delta$ is the only root of height $h$ [Kac 1990, Proposition 6.3]. We find that every monomial corresponding to such a word is zero, and this concludes the proof of the theorem.

\section{The parameter space and generic Lie algebras}

So far we have only considered the Lie algebras $\mathscr{L}(0)$. Now we will be concerned with the variety $X$ of all parameters $f \in\left(\mathscr{F}^{*}\right)^{\Pi}$ for which $\operatorname{dim} \mathscr{L}(f)=\operatorname{dim} \mathscr{L}(0)$. We collect some tools for determining $X$ in the case of simply laced Dynkin diagrams.

4.1. Scaling. First let $\Gamma$ be arbitrary again, not necessarily a Dynkin diagram. Scaling of the generators $x_{i}$ has an effect on $X$ : Given $t=\left(t_{x}\right)_{x \in \Pi}$ in the torus $T:=\left(K^{*}\right)^{\Pi}$, there is a unique automorphism of $\mathscr{F}$ that sends $x \in \Pi$ to $t_{x} x$. This gives an action of $T$ on $\mathscr{F}$, and we endow $\mathscr{F}^{*}$ with the contragredient action. Finally, we obtain an action of $T$ on $X$ by

$$
(t f)_{x}(y):=t_{x}^{-1} f_{x}\left(t^{-1} y\right) \quad \text { for all } t \in T, f \in X, x \in \Pi \text {, and } y \in \mathscr{F} .
$$

Indeed, note that with this definition the automorphism of $\mathscr{F}$ induced by $t$ sends $x x y-f_{x}(y) x \in \mathscr{F}$ to

$$
\begin{aligned}
(t x)(t x)(t y)-f_{x}(y) t x & =t_{x}^{2}\left(x x(t y)-t_{x}^{-1} f_{x}(y) x\right) \\
& =t_{x}^{2}\left(x x(t y)-t_{x}^{-1} f_{x}\left(t^{-1}(t y)\right) x\right) \\
& =t_{x}^{2}\left(x x(t y)-(t f)_{x}(t y) x\right),
\end{aligned}
$$


and hence the ideal $\mathscr{I}(f)$ defining $\mathscr{L}(f)$ to $\mathscr{I}(t f)$. Therefore, this automorphism of $\mathscr{F}$ induces an isomorphism $\mathscr{L}(f) \rightarrow L(t f, \Gamma)$.

This scaling action of $T$ on $X$ will make things very easy in the case of simply laced Dynkin diagrams, where $X$ will turn out to be isomorphic to an affine space with linear action of $T$, in which the maximal-dimensional orbits have codimension 0,1 or 2 .

Remark 15. Observe that the one-parameter subgroup $t \mapsto \lambda(t):=(t, \ldots, t) \in T$ satisfies $\lim _{t \rightarrow \infty} \lambda(t) f=0$ for all $f \in X$. This shows that all irreducible components of $X$ contain 0 ; in particular, $X$ is connected.

4.2. The extremal form. Cohen et al. [2001] proved that on any Lie algebra over a field of characteristic unequal to 2 that is generated by finitely many extremal elements, there is a unique bilinear form $\kappa$ such that $x x y=\kappa(x, y) x$ for all extremal $x$. Moreover, it is shown there that $\kappa$ is symmetric and associative: $\kappa(x, y)=\kappa(y, x)$ and $\kappa(x y, z)=\kappa(x, y z)$ for all $x, y, z$. We call $\kappa$ the extremal form. For the Chevalley algebra $\mathfrak{g}$ of Section 3.2, the extremal form is nonzero on $\mathfrak{g}_{\alpha} \times \mathfrak{g}_{\beta}$ if and only if $\alpha=-\beta$. The form may have a radical contained in the Cartan subalgebra $\mathfrak{h}$.

On the other hand, for any $f \in\left(\mathscr{F}^{*}\right)^{\Pi}$ (not necessarily in $X$ ) the Lie algebra $\mathscr{L}(f)$ is generated by the images of the elements of $\Pi$, which are extremal elements. In particular, for $x \in \Pi$ we have in $\mathscr{L}(f)$

$$
f_{x}(y) x=x x y=\kappa(x, y) x,
$$

where $\kappa$ is the extremal form on $\mathscr{L}(f)$. So if the image of $x$ in $\mathscr{L}(f)$ is nonzero, then $f_{x}(y)=\kappa(x, y)$.

4.3. The Premet relations. Our arguments showing that certain monomials $m:=$ $x_{d} \cdots x_{1}$ are zero in the sandwich algebra $\mathscr{L}(0)$ always depended on the sandwich properties: $x x y=0$ and $x y x z=0$ whenever $x$ is a sandwich element and $y$ and $z$ are arbitrary elements of the Lie algebra. The Premet relations of the following lemma translate such a statement into the following statement: in $\mathscr{L}(f)$ the monomial $\mathrm{m}$ can be expressed in terms of monomials of degree less than $d-1$ and values of $f_{x_{d}}$ on monomials of degree less than $d-1$.

Lemma 16 [Chernousov 1989]. Let $x$ be a nonzero extremal element of a Lie algebra $\mathscr{L}$, and let $f_{x}: \mathscr{L} \rightarrow K$ be the linear function with $x x y=f_{x}(y) x$. Then we have

$$
2 x y x z=f_{x}(y z) x-f_{x}(z) x y-f_{x}(y) x z .
$$

Remark 17. In characteristic 2 the definition of an extremal element $x$ involves the existence of a function $g_{x}$ such that $x y x z=g_{x}(y z) x-g_{x}(z) x y-g_{x}(y) x z$, that is, $g_{x}$ plays the role of $f_{x} / 2$. See [Cohen and Ivanyos 2006, Definition 14]. 
4.4. The parameters. Recall from Section 2 that the restriction map $X \rightarrow\left(V^{*}\right)^{\Pi}$ is injective and has a closed image; a key step in the proof was showing that for $f \in X$ the values $f_{x}(u)$ for $x \in \Pi$ and $u \in F$ depend polynomially on $\left.f\right|_{V}$. In what follows, this will be phrased informally as $f$ can be expressed in $\left.f\right|_{V}$ or $\left.f\right|_{V}$ determines $f$. In this phrase we implicitly assume that $f \in X$, that is, that $\mathscr{L}(f)$ has the maximal possible dimension. In the case of Dynkin diagrams, we will exhibit a small number of values of $f$ in which $f$ can be expressed. For this purpose, the following lemma, which also holds for other graphs, is useful.

Lemma 18. Let $q=x_{d} \cdots x_{1}$ be a monomial of degree $d \geq 2$ and weight $\beta$, and let $z \in \Pi$ be such that $\left\langle\alpha_{z}, \beta\right\rangle \geq-1$. Then $f_{z}(q)$ can be expressed in the parameters $f_{x}(m)$ with monomials $m$ of degree less than $d-1$ and $x \in \Pi$.

Proof. First, if $x_{d}$ is not a $\Gamma$-neighbor of $z$ in $\Gamma$, then

$$
f_{z}\left(x_{d} \cdots x_{1}\right)=\kappa\left(z, x_{d} \cdots x_{1}\right)=-\kappa\left(x_{d} z, x_{d-1} \cdots x_{1}\right)=\kappa\left(0, x_{d-1} \cdots x_{1}\right)=0,
$$

and we are done. So assume that $x_{d}$ is a $\Gamma$-neighbor of $z$. Now

$$
\begin{aligned}
f_{z}\left(x_{d} \cdots x_{1}\right) & =-\kappa\left(x_{d} z, x_{d-1} \cdots x_{1}\right)=\kappa\left(z x_{d}, x_{d-1} \cdots x_{1}\right) \\
& =-f\left(x_{d}, z x_{d-1} \cdots x_{1}\right)=f_{x_{d}}\left(z x_{d-1} \cdots x_{1}\right) .
\end{aligned}
$$

In both cases we have used that the images of $z$ and $x_{d}$ are nonzero in $\mathscr{L}(f)$ for $f \in X$; see Section 4.2. Now $\left\langle\alpha_{z}, \beta-\alpha_{x_{d}}\right\rangle \geq 0$, so Lemma 7 says that $z x_{d-1} \cdots x_{1}$ can be expressed in terms of smaller monomials and values $f_{x}(m)$ for $x \in \Pi$ and monomials $m$ of degree less than $d-1$. Then by linearity of $f_{x_{d}}$, the last expression above can also be expressed in terms of values $f_{x}(m)$ with $x \in \Pi$ and $m$ of degree less than $d-1$.

4.5. Simply laced Dynkin diagrams of finite type. Suppose that $\Gamma$ is a simply laced Dynkin diagram of finite type. Let $\mathfrak{g}$ be the Chevalley algebra with Dynkin diagram $\Gamma$ of Section 3.2. We identify $\mathbb{Z}^{\Pi}$ with the character group of $T=\left(K^{*}\right)^{\Pi}$ in the natural way: we write $\mu$ for the character that sends $t$ to $t^{\mu}=\prod_{x \in \Pi} t_{x}^{\mu_{x}}$. Also let $\Sigma$ be the set of edges of $\Gamma$, and write $\alpha_{e}:=\alpha_{x}+\alpha_{y}$ for $e=\{x, y\} \in \Sigma$.

Theorem 19. Let $\Gamma=(\Pi, \Sigma)$ be a simply laced Dynkin diagram of finite type, obtained from a diagram in Figure 1 by removing vertex 0 . Let $\mathfrak{g}$ be the Chevalley algebra of type $\Gamma$ over the field $K$ of characteristic unequal to 2 . Set $T:=\left(K^{*}\right)^{\Pi}$. Then the variety $X$ is, as a $T$-variety, isomorphic to the vector space $V:=K^{\Sigma}$ on which $T$ acts diagonally with character $-\alpha_{e}$ on the component corresponding to $e \in \Sigma$. For $f$ corresponding to any element in the dense T-orbit $\left(K^{*}\right)^{\Sigma}$, the Lie algebra $\mathscr{L}(f)$ is isomorphic to a fixed Lie algebra.

We first need a lemma that will turn out to describe the generic $\mathscr{L}(f)$. We retain the notation $E_{x}^{0}, H_{x}^{0}, F_{x}^{0} \in \mathfrak{g}$ and $\mathfrak{n}_{+}$from Section 3.2. We denote by $C$ the variety 


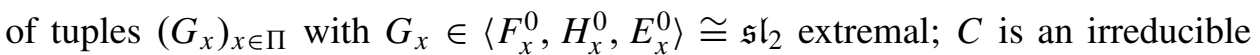
variety.

Lemma 20. For generic $G=\left(G_{x}\right)_{x \in \Pi} \in C$, the Lie subalgebra $\mathfrak{g}^{\prime}$ of $\mathfrak{g}$ generated by the $G_{x}$ has dimension $\operatorname{dim} \mathfrak{n}_{+}$, and moreover $G_{x} G_{x} G_{y}$ is a nonzero multiple of $G_{x}$ for all $x \sim y$.

Proof. By definition $\mathfrak{g}^{\prime}$ is generated by extremal elements; hence it has dimension at most that of $\mathscr{L}(0)$, which is isomorphic to $\mathfrak{n}_{+}$by Theorem 12. The condition that the $G_{x}$ generate a Lie algebra of dimension less than $\operatorname{dim} \mathfrak{n}_{+}$is closed, and the tuple $\left(E_{x}^{0}\right)_{x \in \Pi} \in C$ does not fulfill it. Hence using the irreducibility of $C$, we find that for $G$ in an open dense subset of $C$, the Lie algebra $\mathfrak{g}^{\prime}$ has $\operatorname{dim} \mathfrak{g}^{\prime}=\operatorname{dim} \mathfrak{n}_{+}$. This proves the first statement. The second statement follows directly from the same statement for $\Gamma$ of type $A_{2}$, that is, for $\mathfrak{g}=\mathfrak{s l}_{3}$, where it boils down to the statement that the two copies of $\mathfrak{s l}_{2}$ in $\mathfrak{s l}_{3}$ corresponding to the simple roots are not mutually perpendicular relative to the extremal form in $\mathfrak{s l}_{3}$.

Proof of Theorem 19. By Lemma 18 and Theorem 12, any $f \in X$ is determined by its values $f_{x}(m)$ with $x \in \Pi$ and monomials $m$ of weights $\beta \in \Phi_{+}$such that either $\beta$ has height 1 or $\left\langle\alpha_{x}, \beta\right\rangle \leq-2$. But since $\beta$ is a positive root, the latter inequality cannot hold. Hence $\beta$ has height 1 , so that $m \in \Pi$, and the only $x \in \Pi$ for which $f_{x}(m) \neq 0$ are the neighbors of $m$. Moreover, from the symmetry of the extremal form we conclude that $f_{x}(y)=f_{y}(x)$ for $x, m \in \Pi$ neighbors in $\Gamma$.

We thus find a closed embedding $\Psi: X \rightarrow K^{\Sigma}$ sending $f$ to $\left(f_{x}(y)\right)_{\{x, y\} \in \Sigma}$. Now if we let $T$ act on $K^{\Sigma}$ through the homomorphism

$$
T \rightarrow\left(K^{*}\right)^{\Sigma}, t \mapsto\left(t_{x}^{-1} t_{y}^{-1}\right)_{\{x, y\} \in \Sigma},
$$

then $\Psi$ is $T$-equivariant by the results of Section 4.1. Note that $T$ acts by the character $-\alpha_{e}$ on the component corresponding to $e \in \Sigma$. The fact that $\Gamma$ is a tree readily implies that the characters $\alpha_{e}$ for $e \in \Sigma$ are linearly independent over $\mathbb{Z}$ in the character group of $T$, so that the homomorphism $T \rightarrow\left(K^{*}\right)^{\Sigma}$ is surjective. But then $T$ has finitely many orbits on $K^{\Sigma}$, namely those of the form $\left(K^{*}\right)^{\Sigma^{\prime}} \times\{0\}^{\Sigma \backslash \Sigma^{\prime}}$ with $\Sigma^{\prime} \subseteq \Sigma$.

Now as $\Psi(X)$ is a closed $T$-stable subset of $K^{\Sigma}$, we are done if we can show that $\left(K^{*}\right)^{\Sigma} \cap \Psi(X)$ is nonempty. But this is precisely what Lemma 20 tells us: there exist Lie algebras $\mathfrak{g}^{\prime}$ generated by extremal elements that have the largest possible dimension, and where all coordinates $f_{x}(y)$ with $x \sim y$ are nonzero. This concludes the proof.

Remark 21. The proof above also implies that all Lie algebras described in Lemma 20 are isomorphic. More generally, for any two Lie algebras $\mathfrak{g}^{\prime}$ and $\mathfrak{g}^{\prime \prime}$ with tuples of distinguished, extremal generators $\left(G_{x}^{\prime}\right)_{x \in \Pi}$ and $\left(G_{x}^{\prime \prime}\right)_{x \in \Pi}$ such that 
(i) $G_{x}^{\prime} G_{y}^{\prime}=0$ and $G_{x}^{\prime \prime} G_{y}^{\prime \prime}=0$ for $x \nsim y$,

(ii) $G_{x}^{\prime} G_{x}^{\prime} G_{y}^{\prime}=0$ if and only if $G_{x}^{\prime \prime} G_{x}^{\prime \prime} G_{y}^{\prime \prime}=0$ for $x \sim y$, and

(iii) $\operatorname{dim} \mathfrak{g}^{\prime}=\operatorname{dim} \mathfrak{g}^{\prime \prime}=\operatorname{dim} \mathfrak{n}_{+}$,

there exists an isomorphism $\mathfrak{g}^{\prime} \rightarrow \mathfrak{g}^{\prime \prime}$ mapping each $G_{x}^{\prime}$ to a scalar multiple of $G_{x}^{\prime \prime}$.

4.6. Simply laced Dynkin diagrams of affine type. Suppose now that $\Gamma$ is a simply laced Dynkin diagram of affine type. We retain the notation from Section 3.3. In particular, let $\mathfrak{g}$ be the Chevalley algebra of type $\Gamma^{0}$, the graph induced by $\Gamma$ on $\Pi^{0}=\Pi \backslash\left\{x_{0}\right\}$. To state the analogue of Theorem 19 , we again identify $\mathbb{Z}^{\Pi}$ with the character group of $T=\left(K^{*}\right)^{\Pi}$ and retain the notation $\alpha_{e}$ for $e \in \Sigma$, the edge set of $\Gamma$.

Theorem 22. Let $\Gamma=(\Pi, \Sigma)$ be a simply laced Dynkin diagram of affine type from Figure 1, and let $\Gamma^{0}$ be the finite-type diagram obtained by deleting vertex 0 from $\Gamma$. Let $\mathfrak{g}$ be the Chevalley algebra of type $\Gamma^{0}$ over the field $K$ of characteristic unequal to 2 , and set $T:=\left(K^{*}\right)^{\Pi}$. Then the variety $X$ is, as a $T$-variety, isomorphic to the vector space $V:=K^{\Sigma} \times K$ on which $T$ acts diagonally with character $-\alpha_{e}$ on the component corresponding to $e \in \Sigma$, and with character $-\delta$ on the last component. For all $f \in X$ corresponding to points in some open dense subset of $K^{\Sigma} \times K$, the Lie algebra $\mathscr{L}(f)$ is isomorphic to $\mathfrak{g}$.

Remark 23. Unlike for diagrams of finite type, it is not necessarily true that $T$ has only finitely many orbits on $V$. Indeed, the following three situations occur:

(i) The characters $\alpha_{e}$ (for $e \in \Sigma$ ) and $\delta$ are linearly independent. This is the case for $D_{\text {even }}^{(1)}, E_{7}^{(1)}$, and $E_{8}^{(1)}$. Then $T$ has finitely many orbits on $V$.

(ii) The characters $\alpha_{e}$ for $e \in \Sigma$ are linearly independent, but $\delta$ is in their $\mathbb{Q}$ linear span. This is the case for $A_{\text {even }}^{(1)}, D_{\text {odd }}^{(1)}$ and $E_{6}^{(1)}$. Now the orbits of $T$ in $\left(K^{*}\right)^{\Sigma} \times K^{*}$ have codimension 1 . The character $\delta$, for $A_{\text {even }}^{(1)}$ and $E_{6}^{(1)}$, has full support when expressed in the $\alpha_{e}$; this readily implies that $T$ has finitely many orbits on the complement of $\left(K^{*}\right)^{\Sigma} \times K^{*}$. For $D_{n}^{(1)}$ with $n$ odd, however, $(n-3) / 2$ edge characters get coefficient 0 when $\delta$ is expressed in them, and therefore $T$ still has infinitely many orbits on said complement.

(iii) The characters $\alpha_{e}$ for $e \in \Sigma$ are linearly dependent. This is the case only for $A_{\mathrm{odd}}^{(1)}$, and in fact $\delta$ is then also in the span of the $\alpha_{e}$. Now the $T$-orbits in $\left(K^{*}\right)^{\Sigma} \times K^{*}$ have codimension 2 , and in the complement there are still infinitely many orbits.

This gives some feeling for the parameter space $X$. It would be interesting to determine exactly all isomorphism types of Lie algebras $\mathscr{L}(f)$ with $f \in X$ - but here we confine ourselves to those with $f$ in some open dense subset of $K^{\Sigma} \times K$. 
The proof is very similar to that of Theorem 19. Again, we first prove a lemma that turns out to describe the generic $\mathscr{L}(f)$. Retain the notation $E_{x}^{0}, H_{x}^{0}, F_{x}^{0} \in \mathfrak{g}$ for $x \in \Pi^{0}$. Denote the lowest weight by $\theta \in \Phi_{-}^{0}$, let $E_{x_{0}}^{0}$ and $F_{x_{0}}^{0} \in \mathfrak{g}$ be the elements of the Chevalley basis of weights $\theta$ and $-\theta$, respectively, and set $H_{x_{0}}^{0}:=\left[E_{x_{0}}^{0}, F_{x_{0}}^{0}\right]$. Write $C$ for the irreducible variety of tuples $\left(G_{x}\right)_{x \in \Pi}$ with $G_{x} \in\left\langle F_{x}^{0}, H_{x}^{0}, E_{x}^{0}\right\rangle \cong \mathfrak{s l}_{2}$ extremal.

Lemma 24. For generic $G=\left(G_{x}\right)_{x \in \Pi} \in C$, the $G_{x}$ generate $\mathfrak{g}$, and $G_{x} G_{x} G_{y}$ is a nonzero multiple of $G_{x}$ for all $x \sim y$.

Proof. The first statement is true for $G=\left(E_{x}^{0}\right)_{x \in \Pi}$; this follows from the properties of the Chevalley basis in Section 3.2. Hence by the irreducibility of $C$ it is true generically. The second statement follows, as in Lemma 20, from the same statement in $\mathfrak{s l}_{3}$.

In the following proof we will show that the choice of $\left(G_{x}\right)_{x \in \Pi}$ as in Lemma 24 already gives generic points in $X$, except for the case where $\Gamma$ is of type $A_{\mathrm{odd}}^{(1)}$, for which we give another construction.

Proof of Theorem 22. By Lemma 18 and Theorem 13, any $f \in X$ is determined by its values $f_{x}(m)$ with $x \in \Pi$ and monomials $m$ of weights $\beta \in \Theta$ such that either $\beta$ has height 1 or $\left\langle\alpha_{x}, \beta\right\rangle \leq-2$. In contrast with the case of finite-type diagrams, there do exist pairs $(x, \beta) \in \Pi \times \Theta$ with this latter property, namely, precisely those of the form $\left(x, \delta-\alpha_{x}\right)$. For all $x \in \Pi$, let $m_{x}$ be a monomial that spans the weight space in $\mathscr{L}(0)$ of weight $\delta-\alpha_{x}$; this space is 1-dimensional by Theorem 13. We claim that the $f_{x}\left(m_{x}\right)$ can all be expressed in terms of $f_{x_{0}}\left(m_{x_{0}}\right)$ and values $f_{z}(r)$ with $z \in \Pi$ and $r$ of degree less than $h-2$. Indeed, if $x \neq x_{0}$, then $x_{0}$ occurs exactly once in $m_{x}$; and writing $m_{x}=x_{d} \cdots x_{1} x_{0} y_{e} \cdots y_{1}$ with $x_{1}, \ldots, x_{d}, y_{1}, \ldots, y_{e} \in \Pi^{0}$, we find

$$
\begin{aligned}
f_{x}\left(m_{x}\right) & =\kappa\left(x, x_{d} \cdots x_{1} x_{0} y_{e} \cdots y_{1}\right) \\
& =(-1)^{d+1} \kappa\left(x_{0} x_{1} \cdots x_{d} x, y_{e} \cdots y_{1}\right) \\
& =(-1)^{d} \kappa\left(x_{0},\left[x_{1} \cdots x_{d} x, y_{e} \cdots y_{1}\right]\right) \\
& =(-1)^{d} f_{x_{0}}\left(\left[x_{1} \cdots x_{d} x, y_{e} \cdots y_{1}\right]\right),
\end{aligned}
$$

and the expression $\left[x_{1} \cdots x_{d} x, y_{e} \cdots y_{1}\right]$ can be rewritten in terms of $m_{x_{0}}$ and shorter monomials, using values $f_{z}(r)$ with $r$ of degree less than $d+e=h-2$.

We have now found a closed embedding $X \rightarrow K^{\Sigma} \times K$ which sends $f$ to $\left(\left(f_{x}(y)\right)_{\{x, y\} \in \Sigma}, f_{x_{0}}\left(m_{x_{0}}\right)\right)$; for ease of exposition we will view $X$ as a closed subset of $K^{\Sigma} \times K$. The theorem follows once we can realize generic parameter values in $K^{\Sigma} \times K$ with extremal elements that generate $\mathfrak{g}$. To this end, choose a generic tuple $\left(G_{x}\right)_{x \in \Pi}$ in $C$. By Lemma 24 these generate $\mathfrak{g}$, and they clearly satisfy $G_{x} G_{y}=0$ for $x \not y$. Hence they yield a point in $X$ with $f_{x}(y)=\kappa\left(G_{x}, G_{y}\right) \neq 0$ for $x \sim y$. Furthermore, the parameter $f_{x_{0}}\left(m_{x_{0}}\right)$ equals the extremal form evaluated on $G_{x_{0}}$ 
and the monomial $m_{x_{0}}$ evaluated in the $G_{x}$. Express that monomial in the $G_{x}$ as $\xi E_{x_{0}}^{0}+\eta H_{x_{0}}^{0}+\zeta F_{x_{0}}^{0}$ plus a term perpendicular to $\left\langle E_{x_{0}}^{0}, H_{x_{0}}^{0}, F_{x_{0}}^{0}\right\rangle$, and write $G_{x_{0}}$ as $\xi^{\prime} E_{x_{0}}^{0}+\eta^{\prime} H_{x_{0}}^{0}+\zeta^{\prime} F_{x_{0}}^{0}$. For the degenerate case where $G_{x}=E_{x}^{0}$ for all $x$, we have $\xi, \eta=0$ and $\zeta \neq 0$ (that monomial is a nonzero scalar multiple of the highest root vector, $\left.F_{x_{0}}^{0}\right)$, so that $f_{x_{0}}\left(m_{x_{0}}\right)=\kappa\left(E_{x_{0}}^{0}, \zeta F_{x_{0}}^{0}\right) \neq 0$. Therefore, this parameter is nonzero generically. Hence we have found a point $f \in X \bigcap\left(\left(K^{*}\right)^{\Sigma} \times K^{*}\right)$. Along the lines of Remark 23 we now distinguish three cases:

(i) If the $\alpha_{e}$ (for $e \in \Sigma$ ) and $\delta$ are linearly independent, then $T$ acts transitively on $\left(K^{*}\right)^{\Sigma} \times K^{*}$, and we are done.

(ii) If the $\alpha_{e}$ for $e \in \Sigma$ are linearly independent, but $\delta$ lies in their span, then we now show that we can alter the point $f$ above in a direction transversal to its $T$-orbit. Let $S$ be the torus in the adjoint group of $\mathfrak{g}$ whose Lie algebra is $\mathfrak{h}$, and consider the effect on $f$ of conjugation of $G_{x_{0}}$ with an element $s \in S$, while keeping the other $G_{x}$ fixed. This transforms $G_{x_{0}}=\xi^{\prime} E_{x_{0}}^{0}+\eta^{\prime} H_{x_{0}}^{0}+\zeta^{\prime} F_{x_{0}}^{0}$ in $s^{\theta} \xi^{\prime} E_{x_{0}}^{0}+\eta^{\prime} H_{x_{0}}^{0}+s^{-\theta} \zeta^{\prime} F_{x_{0}}$, and therefore it transforms $f_{x_{0}}\left(m_{x_{0}}\right)$ into

$$
s^{\theta} \xi^{\prime} \zeta \kappa\left(E_{x_{0}}^{0}, F_{x_{0}}^{0}\right)+\eta^{\prime} \eta \kappa\left(H_{x_{0}}^{0}, H_{x_{0}}^{0}\right)+s^{-\theta} \zeta^{\prime} \xi \kappa\left(F_{x_{0}}^{0}, E_{x_{0}}^{0}\right)
$$

while it keeps the parameters $f_{x_{0}}(y)$ with $x_{0} \sim y$ unchanged; these only depend on $\eta^{\prime}$. This shows that we can indeed move $f$ inside $X$ in a direction transverse to its $T$-orbit, and we are done.

(iii) Finally, in the case of $A_{n-1}^{(1)}$ with $n$ even, we first show that tuples in $C$ only give points in a proper closed subset of $K^{\Sigma} \times K$. Here $\mathfrak{g}=\mathfrak{s l}_{n}$ and $\Gamma$ is an $n$ cycle; label its points $0, \ldots, n-1$. Relative to the usual choices of $E_{i}^{0}, H_{i}^{0}, F_{i}^{0}$, the element $G_{i}$ is a matrix with $2 \times 2$-block

$$
\left[\begin{array}{cc}
a_{i} b_{i} & a_{i}^{2} \\
-b_{i}^{2} & -a_{i} b_{i}
\end{array}\right]
$$

on the diagonal in rows (and columns) $i$ and $i+1$ and with zeros elsewhere. We count the rows and columns modulo $n$ so that row 0 is actually row $n$. But then we have $\kappa\left(G_{i}, G_{i+1}\right)=2 a_{i} b_{i} a_{i+1} b_{i+1}$, and this implies

$$
\begin{aligned}
\kappa\left(G_{1}, G_{2}\right) & \kappa\left(G_{3}, G_{4}\right) \cdots \kappa\left(G_{n-1}, G_{0}\right) \\
& =2^{n}\left(a_{1} b_{1}\right)\left(a_{2} b_{2}\right)\left(a_{3} b_{3}\right)\left(a_{4} b_{4}\right) \cdots\left(a_{n-1} b_{n-1}\right)\left(a_{0} b_{0}\right) \\
& =2^{n}\left(a_{0} b_{0}\right)\left(a_{1} b_{1}\right)\left(a_{2} b_{2}\right)\left(a_{3} b_{3}\right) \cdots\left(a_{n-2} b_{n-2}\right)\left(a_{n-1} b_{n-1}\right) \\
& =\kappa\left(G_{0}, G_{1}\right) \kappa\left(G_{2}, G_{3}\right) \cdots \kappa\left(G_{n-2}, G_{n-1}\right) ;
\end{aligned}
$$

so the tuple of parameter values of the tuple $\left(G_{i}\right)_{i=0}^{n-1} \in C$ lies in a proper closed subset $R$ of $K^{\Sigma} \times K$. 
We therefore allow the tuple $\left(G_{i}\right)_{i=0}^{n-1}$ to vary in a slightly larger variety $C^{\prime} \supset C$ as follows: the conditions on $G_{1}, \ldots, G_{n-1}$ remain the same, but $G_{0}$ is now allowed to take the shape

$$
\left[\begin{array}{ccccc}
-a_{0} b_{0} & 0 & \ldots & 0 & -b_{0}^{2} \\
c_{2} a_{0} & 0 & \ldots & 0 & c_{2} b_{0} \\
\vdots & \vdots & & \vdots & \vdots \\
c_{n-1} a_{0} & 0 & \ldots & 0 & c_{n-1} b_{0} \\
a_{0}^{2} & 0 & \ldots & 0 & a_{0} b_{0}
\end{array}\right]
$$

(which is extremal since it has rank 1 and trace 0 ), subject to the equations

$$
b_{i} c_{i}+a_{i} c_{i+1}=0 \quad \text { for } i=2, \ldots, n-2,
$$

which ensure that $G_{0}$ commutes with $G_{2}, \ldots, G_{n-2}$. Still, any tuple in an open neighborhood $U \subseteq C^{\prime}$ of our original tuple $\left(G_{i}\right)_{i=0}^{n-1}$ (with generic $a_{i}$ and $b_{i}$ but all $c_{i}$ equal to 0$)$ generates $\mathfrak{s l}_{n}$. We now argue that the differential $d$ at $\left(G_{i}\right)_{i=0}^{n-1}$ of the map $U \rightarrow X \subseteq K^{\Sigma} \times K$ sending a tuple to the parameters that it realizes has rank $|\Sigma|+1$, as required. Indeed, the $T$-action already gives a subspace of dimension $|\Sigma|-1$, tangent to $R$. Making $c_{2}$ (and hence all $c_{i}$ ) nonzero adds $-2 a_{1}^{2} c_{2} a_{0}$ to $\kappa\left(G_{0}, G_{1}\right)$ and $2 b_{n-1}^{2} c_{n-1} b_{0}$ to $\kappa\left(G_{0}, G_{n-1}\right)$, and it fixes all other $\kappa\left(G_{i}, G_{j}\right)$. We show that this infinitesimal direction is not tangent to $R$ : it adds

$$
2^{n} b_{n-1}^{2} c_{n-1} b_{0}\left(a_{1} b_{1}\right)\left(a_{2} b_{2}\right) \cdots\left(a_{n-3} b_{n-3}\right)\left(a_{n-2} b_{n-2}\right)
$$

to the left side of (2), and

$$
-2^{n} a_{1}^{2} c_{2} a_{0}\left(a_{2} b_{2}\right)\left(a_{3} b_{3}\right) \cdots\left(a_{n-2} b_{n-2}\right)\left(a_{n-1} b_{n-1}\right)
$$

to the right side. Dividing these expressions by common factors, the first becomes $2^{n} b_{n-1} c_{n-1} b_{0} b_{1}$ and the second $-2^{n} a_{1} c_{2} a_{0} a_{n-1}$. These expressions are not equal generically, even modulo the equations (3) relating the $c_{i}$ to the $a_{i}$ and $b_{i}$; indeed, these equations do not involve $a_{0}, a_{1}, a_{n-1}, b_{0}, b_{1}, b_{n-1}$.

Note that varying $c_{2}$ may also effect the parameter $f_{x_{0}}\left(m_{x_{0}}\right)$, but in any case the above shows that the composition of the differential $d$ with projection onto $K^{\Sigma}$ is surjective. On the other hand, conjugation with the torus $S$ as in case (ii) yields a vector in the image of $d$ which is supported only on the factor $K$ corresponding to $\delta$. This concludes the proof that $d$ has full rank.

\section{Notes}

5.1. Recognizing the simple Lie algebras. Going through the proof that $X$ is an affine variety, one observes that the map $\left.f \mapsto f\right|_{V}$ is not only injective on $X$, but 
even on

$$
X^{\prime}(\Gamma):=\left\{f \in\left(\mathscr{F}^{*}\right)^{\Pi} \mid \text { for all } x \in \Pi: x \neq 0 \text { in } \mathscr{L}(f)\right\} \supseteq X .
$$

The same is true for the map $f \mapsto\left(\left(f_{x}(y)\right)_{\{x, y\} \in \Sigma}\right)$ in the case where $\Gamma$ is a Dynkin diagram of finite type, and for the map $f \mapsto\left(\left(f_{x}(y)\right)_{\{x, y\} \in \Sigma}, f_{x_{0}}\left(m_{x_{0}}\right)\right)$ in the case where $\Gamma$ is a Dynkin diagram of affine type. This shows that, for these Dynkin diagrams, $X^{\prime}(\Gamma)$ is actually equal to $X$, whence the following theorem.

Theorem 25. Suppose that $\Gamma$ is a Dynkin diagram of finite or affine type. Let $\mathscr{L}$ be any Lie algebra, over a field of characteristic unequal to 2 , which is generated by nonzero extremal elements $G_{x}$ for $x \in \Pi$ in which the commutation relations $G_{x} G_{y}=0$ hold for $x \ngtr y$. Define $f \in\left(\mathscr{F}^{*}\right)^{\Pi}$ by the condition that $G_{x} G_{x} u=$ $f_{x}(u) G_{x}$ holds in $\mathscr{L}$. Then $f \in X$ and $\mathscr{L}$ is a quotient of $\mathscr{L}(f)$.

This theorem could well prove useful for recognizing the Chevalley algebras $\mathfrak{g}$ : if $f$ corresponds to a point in the open dense subset of $K^{\Sigma} \times K$ referred to in Theorem 22 , then one concludes that $\mathscr{L}$ is a quotient of $\mathfrak{g}$. Hence if $\mathfrak{g}$ is a simple Lie algebra, then $\mathscr{L}$ is isomorphic to $\mathfrak{g}$. It is not clear to us whether, for general $\Gamma$, the image of $X^{\prime}(\Gamma)$ in $\left(V^{*}\right)^{\Pi}$ is closed; this is why we chose to work with $X$ instead.

5.2. Other graphs. Our methods work very well for Dynkin diagrams, but for more general graphs new ideas are needed to determine $\mathscr{L}(0), X$, and $\mathscr{L}(f)$ for $f \in X$. The relation with the Kac-Moody algebra of $\Gamma$ may be much tighter than we proved in Section 3.1. General questions of interest are: Is $X$ always an affine space? Is there always a generic Lie algebra? We expect the answers to both questions to be negative, but do not have any counterexamples.

The references [in 't panhuis et al. 2007; Postma 2007; Roozemond 2005] contain other series of graphs which exhibit the same properties as we have proved here: the variety $X$ is an affine space, and generic points in it correspond to simple Lie algebras of types $A_{n}, C_{n}, B_{n}, D_{n}$. In fact, the graph that they find for $C_{n}$ is just the finite-type Dynkin diagram of type $A_{2 n}$. This also follows easily from our results: take $2 n$ generic extremal elements $\left(G_{x}\right)_{x}$ in $\mathfrak{s l}_{2 n+1}$ as in Lemma 20. These generate a subalgebra of $\mathfrak{s l}_{2 n+1}$ of dimension $\left(\begin{array}{c}2 n+1 \\ 2\end{array}\right)$ by that same lemma, and if we consider them as matrices, their images span a subspace $W$ of dimension $2 n$ in $K^{2 n+1}$. It is not hard to write down an explicit, nondegenerate skew symmetric form on $W$ with respect to which the $G_{x}$ are skew - hence the Lie algebra generated by them is $\mathfrak{s p}_{2 n}$.

\section{References}

[Billig 1990] Y. V. Billig, "Modular affine Lie algebras", Mat. Sb. 181:8 (1990), 1130-1143. In Russian; translated in Math. USSR-Sb. 70:2 (1991), 573-586. MR 91k:17016 Zbl 0715.17024 
[Carter 1972] R. W. Carter, Simple groups of Lie type, Pure and Applied Mathematics 28, Wiley, London, 1972. MR 53 \#10946 Zbl 0248.20015

[Chernousov 1989] V. I. Chernousov, "The Hasse principle for groups of type E8", Dokl. Akad. Nauk SSSR 306:5 (1989), 1059-1063. In Russian; translated in Soviet Math. Dokl. 39:3 (1989), 592-596. MR 90m:11055 Zbl 0703.20040

[Cohen and Ivanyos 2006] A. M. Cohen and G. Ivanyos, "Root filtration spaces from Lie algebras and abstract root groups", J. Algebra 300:2 (2006), 433-454. MR 2008a:17008 Zbl 1109.51006

[Cohen et al. 2001] A. M. Cohen, A. Steinbach, R. Ushirobira, and D. Wales, "Lie algebras generated by extremal elements", J. Algebra 236:1 (2001), 122-154. MR 2001k:17007 Zbl 1076.17500

[Kac 1990] V. G. Kac, Infinite-dimensional Lie algebras, 3rd ed., Cambridge University Press, 1990. MR 92k:17038 Zbl 0716.17022

[Kostrikin 1981] A. I. Kostrikin, "Sandwiches in Lie algebras", Math. USSR, Sb. 38 (1981), 1-9. Zbl 0449.17003

[in 't panhuis et al. 2007] J. C. H. W. in 't panhuis, E. J. Postma, and D. A. Roozemond, "Extremal presentations for classical Lie algebras", Preprint, 2007. arXiv 0705.2332v3

[Postma 2007] E. J. Postma, From Lie algebras to geometry and back, Thesis, Technische Universiteit Eindhoven, 2007, Available at http://alexandria.tue.nl/extra2/200710440.pdf.

[Premet and Strade 1997] A. Premet and H. Strade, "Simple Lie algebras of small characteristic, I: Sandwich elements”, J. Algebra 189:2 (1997), 419-480. MR 98e:17029 Zbl 0878.17019

[Roozemond 2005] D. A. Roozemond, Lie algebras generated by extremal elements, Master's thesis, Technische Universiteit Eindhoven, 2005, Available at http://alexandria.tue.nl/extra1/afstversl/ wsk-i/roozemond2005.pdf.

[Seligman 1967] G. B. Seligman, Modular Lie algebras, Ergebnisse der Mathematik und ihrer Grenzgebiete 40, Springer, New York, 1967. MR 39 \#6933 Zbl 0189.03201

[Strade 2004] H. Strade, Simple Lie algebras over fields of positive characteristic, I: Structure theory, de Gruyter Expositions in Mathematics 38, de Gruyter, Berlin, 2004. MR 2005c:17025 Zbl 1074.17005

[Zelmanov and Kostrikin 1990] E. I. Zel'manov and A. I. Kostrikin, "A theorem on sandwich algebras”, Trudy Mat. Inst. Steklov. 183 (1990), 106-111, 225. In Russian; translated in Proc. Steklov Inst. Math. 183:4 (1991), 121-126. MR 92h:17007 Zbl 0729.17006

Communicated by Efim Zelmanov

Received 2007-08-17 Revised 2008-03-06 Accepted 2008-05-27

j.draisma@tue.nl

Department of Mathematics and Computer Science, Technische Universiteit Eindhoven, P.O. Box 513, 5600 MB Eindhoven, The Netherlands http://www.win.tue.nl/ jdraisma/

j.c.h.w.panhuis@tue.nl

Department of Mathematics and Computer Science, Technische Universiteit Eindhoven, P.O. Box 513, 5600 MB Eindhoven, The Netherlands http://www.win.tue.nl/ jpanhuis/ 